\title{
Stem Cell Therapy in Dengue Virus-Infected BALB/C Mice Improves Hepatic Injury
}

\author{
S. Sakinah ${ }^{1}$, Sivan Padma Priya ${ }^{1}$, Pooi Ling Mok ${ }^{2,3 *}$, Rusheni Munisvaradass ${ }^{3}$, \\ Seoh Wei Teh', Zhong Sun ${ }^{3}$, Badr Alzahrani' ${ }^{2}$ Faizal Abu Bakar', Hui-yee Chee', \\ Rukman Awang Hamat ${ }^{1}$, Guozhong He ${ }^{5}$, Chenglong Xiong ${ }^{6}$, Narcisse Joseph ${ }^{1}$, \\ Jia Bei Tong ${ }^{1}$, Xiaoyun Wu$^{7}$, Mahendran Maniam ${ }^{8}$, Antony V. Samrot ${ }^{9}$, Akon Higuchi ${ }^{10,11}$ \\ and S. Suresh Kumar ${ }^{1,12 *}$
}

\section{OPEN ACCESS}

Edited by:

Stevo J. Najman,

University of Niš, Serbia

Reviewed by:

Natália Gedeão Salomão, Oswaldo Cruz Foundation (Fiocruz),

Brazil

Ranjith Kumar Rajendran, National Central University, Taiwan Sreekanth Gopinathan Pillai, Indian Institute of Chemical

Technology (CSIR), India Thawornchai Limjindaporn, Mahidol University, Thailand

*Correspondence:

S. Suresh Kumar sureshkudsc@gmail.com

Pooi Ling Mok

mpling@ju.edu.sa/ rachelmok2005@gmail.com

Specialty section:

This article was submitted to Cell Growth and Division,

a section of the journal

Frontiers in Cell and Developmental

Biology

Received: 03 December 2020 Accepted: 25 May 2021

Published: 05 July 2021

Citation:

Sakinah S, Priya SP, Mok PL, Munisvaradass $R$, Teh SW, Sun Z Alzahrani B, Abu Bakar F, Chee H-y, Awang Hamat $R$, He G, Xiong $C$, Joseph N, Tong JB, Wu X, Maniam M, Samrot AV, Higuchi $A$ and Kumar SS (2021) Stem Cell Therapy in Dengue Virus-Infected BALB/C

Mice Improves Hepatic Injury. Front. Cell Dev. Biol. 9:637270. doi: 10.3389/fcell.2021.637270
${ }^{1}$ Department of Medical Microbiology, Faculty of Medicine and Health Sciences, Universiti Putra Malaysia, Seri Kembangan, Malaysia, ${ }^{2}$ Department of Clinical Laboratory Sciences, College of Applied Medical Sciences, Jouf University, Sakakah,

Saudi Arabia, ${ }^{3}$ Department of Biomedical Sciences, Faculty of Medicine and Health Sciences, Universiti Putra Malaysia, Seri Kembangan, Malaysia, ${ }^{4}$ Bioinformatics and Computational Biology, Malaysia Genome Institute, National Institute of Biotechnology Malaysia (NIBM), Kajang, Malaysia, ${ }^{5}$ Institute of Health, Kunming Medical University, Kunming, China, ${ }^{6}$ Department of Medical Microbiology, School of Public Health, Fudan University, Shanghai, China, ${ }^{7}$ First Affiliated Hospital of Baotou Medical College, Inner Mongolia University of Science and Technology, Baotou, China, ${ }^{8}$ Sultan Idris Education University, Tanjong Malim, Malaysia, ${ }^{9}$ School of Bioscience, Faculty of Medicine, Bioscience and Nursing, MAHSA University, Jenjarom, Malaysia, ${ }^{10}$ Department of Chemical and Materials Engineering, National Central University, Taoyuan City, Taiwan, ${ }^{11} R \& D$ Center for Membrane Technology, Chung Yuan Christian University, Taoyuan City, Taiwan, ${ }^{12}$ Centre for Materials Engineering and Regenerative Medicine, Bharath Institute of Higher Education and Research, Chennai, India

Extensive clinical efforts have been made to control the severity of dengue diseases; however, the dengue morbidity and mortality have not declined. Dengue virus (DENV) can infect and cause systemic damage in many organs, resulting in organ failure. Here, we present a novel report showing a tailored stem-cell-based therapy that can aid in viral clearance and rescue liver cells from further damage during dengue infection. We administered a combination of hematopoietic stem cells and endothelial progenitor cells in a DENV-infected BALB/c mouse model and found that delivery of this cell cocktail had improved their liver functions, confirmed by hematology, histopathology, and nextgeneration sequencing. These stem and progenitor cells can differentiate into target cells and repair the damaged tissues. In addition, the regime can regulate endothelial proliferation and permeability, modulate inflammatory reactions, enhance extracellular matrix production and angiogenesis, and secrete an array of growth factors to create an enhanced milieu for cell reparation. No previous study has been published on the treatment of dengue infection using stem cells combination. In conclusion, dengueinduced liver damage was rescued by administration of stem cell therapy, with less apoptosis and improved repair and regeneration in the dengue mouse model.

Keywords: dengue infection, stem cell therapy, DENV 2, next-generation sequencing, hepatology

\section{INTRODUCTION}

Dengue virus (DENV) infections transmitted by mosquitoes is setting half of the world's population at risk. Despite advancement in biomedical sciences in the past decades, dengue morbidity and mortality have not declined. Each year, 390 million people are infected with dengue fever, and 96 million of them have different degrees of clinical manifestations (Bhatt et al., 2013). 
There are currently no specific treatments and substantial vector control measures to prevent their rapid emergence and global spread. As a result, the global incidence of dengue fever has increased dramatically in recent decades, and it is urgent to introduce a new alternative therapy to overcome dengue. Here, we show a novel therapeutic approach using a combination of stem cells [hematopoietic stem cells (HSCs) and endothelial progenitor cells (EPCs)] to treat DENV infection and rescue clinical outcome and dengue-induced liver injury in an animal model. The focal points studied were changes in the state of the animals, clinical profiles, histopathology, and next-generation sequencing (NGS) of each liver gene expressed in the control, DENV-infected (DVI), and stem-cells-treated (DVI-SCT) groups. To that effect, the therapeutic efficacy of stem cells against viral infection was evaluated by observing and examining several physical parameters, including behavioral changes exhibited by the experimental animals, clinical profile parameters (i.e., platelet, white blood cell, red blood cell counts, and lymphocyte, and hemoglobin levels), histopathological changes, and NGS data. The findings demonstrate that the use of combination stem cell therapy cannot only heal vascular injury, thrombocytopenia, and hepatocyte damage caused by DENV but also reduce the presence of DENV in the liver tissue, suggesting clearance of DENV.

\section{RESULTS AND DISCUSSION}

\section{Behavioral Changes in Experimental Animals}

While other mouse models have been genetically modified and/or immunocompromised, we choose BALB/c mouse model, as it would mimic the normal human condition during the occurrence of dengue infection (Barth et al., 2006). The changes in mice observed throughout the experiment are shown in Figure 1. No death was observed throughout the experiment. The weight of each mouse was plotted in Supplementary Figure 4. There was no significant difference in weight of mice in each group. The mice in the control group were active and showed no signs of hemorrhage and bleeding (Figures 1Aj,m), and the condition of the fur was well-groomed throughout the experiment (Figures 1Aa,d,g). Meanwhile, the mice in the DVI group displayed ruffled fur after dengue infection (Figures 1Ae,h). A marginal purple-bluish spot was found under the abdominal skin area and deemed a sign of bleeding in the DVI group mice (Figure 1Ak). They stayed close to each other and had poor mobility and temporary paralysis of the hind limbs (Figure 1An). The above condition continued until the end of the experiment and did not improve significantly.

On the other hand, the mice in the DVI-SCT group developed the same conditions as the DVI group at the early stage of dengue infection, such as inactivity, poor mobility, and temporary paralysis of the hind limbs (Figure 1Ao) but no signs of bleeding (Figure 1Al). The above symptoms recovered to the state similar to the control group mice, within 1 week after stem cell treatment. At the same time, ruffled fur at first day postinfection (dpi) of dengue infection (Figure 1Ac) was observed to be improved
1 day after stem cell treatment (Figure 1Af), then were wellgroomed by the sixth $\mathrm{dpi}$, and remained groomed until the 21st dpi (Figure 1Ai).

Dengue fever can cause muscle pain and muscle weakness, and the mice in the DVI group showed the corresponding poor mobility, hind limb paralysis, and restricted movement. Dengueinduced thrombocytopenia and hemorrhage were also presented as mild bleeding in DVI mice. Furthermore, dengue fever can cause hypovolemic shock, and the phenomenon of ruffled fur due to piloerection, hypothermia, and close proximity to each other in the DVI group is evidenced. After stem cell treatment (SCT), the ruffled fur, paralysis, and mobility all recovered within a few days, and no signs of hemorrhage were observed (Figure 1A). This was likely due to the stem cell treatment, especially with EPCs, which promote endothelial repair and neovascularization, in turn assisting in recovery from paralysis and hemorrhage (McAllister et al., 2013). DENV 2 was inoculated intraperitoneally because we would like to mimic the human dengue-infected signs and also to establish severe dengue infection. Intraperitoneal route establishes signs of dengue diseases even with lower viral dose inoculation including thrombocytopenia, liver damage, hemorrhage, and viremia and disseminated to various organs. In addition, DENV infection of animals by intraperitoneal route also reproduce some aspect of human disease (Paes et al., 2005; Kuruvilla et al., 2007; Zellweger et al., 2017). Moreover, in wild-type mouse models, intraperitoneal DENV-infection results in neurological abnormalities, which is considered as criteria for severe dengue by the World Health Organization (World Health Organization (WHO), 2009). The stem cell treatment was performed via intravenous administration to increase hematopoietic reconstitution in the mice bone marrow. Apart from being the most commonly used technique for intravenous route treatment, it was reported to be safe and had no tumor formations and no cases of infections or increased pain in human trials. In addition, it also helps in rapid diffusion of donor cell population, faster repair of blood vessels, and increase in longterm engraftment without increasing fetal mortality (Geffner et al., 2008; Yang et al., 2015; Boelig et al., 2016).

\section{Clinical Profile Analysis}

Platelet dysfunction is one of the hallmarks of dengue infection, along with pronounced thrombocytopenia. To assess the effects of stem cells treatment on platelets and other blood cells, blood analysis was performed on every group of mice at different dpi (Figure 1B). In this study, the platelet counts in the DVI group were lower than that in the control and DVI-SCT groups. The slight increase on the 15th dpi in the DVI group is suggestive of the body innate immune system trying to recover the platelet counts to a normal level; however, it failed, and the count dropped to a critical level (near zero) by 21 st dpi comparable with the platelet count trend by Frias-Staheli et al. (Jiang et al., 2013). Mice in the DVI-SCT group showed an increase in platelet count from fifth dpi and achieved similar level with that of the control group on 21st dpi (Figure 1Ba). It indicated that the administration of the stem cells cocktails during DENV2 infection has restored platelet count to the normal level. The HSCs in the stem cells cocktail can differentiate into megakaryocytes and stimulate bone 


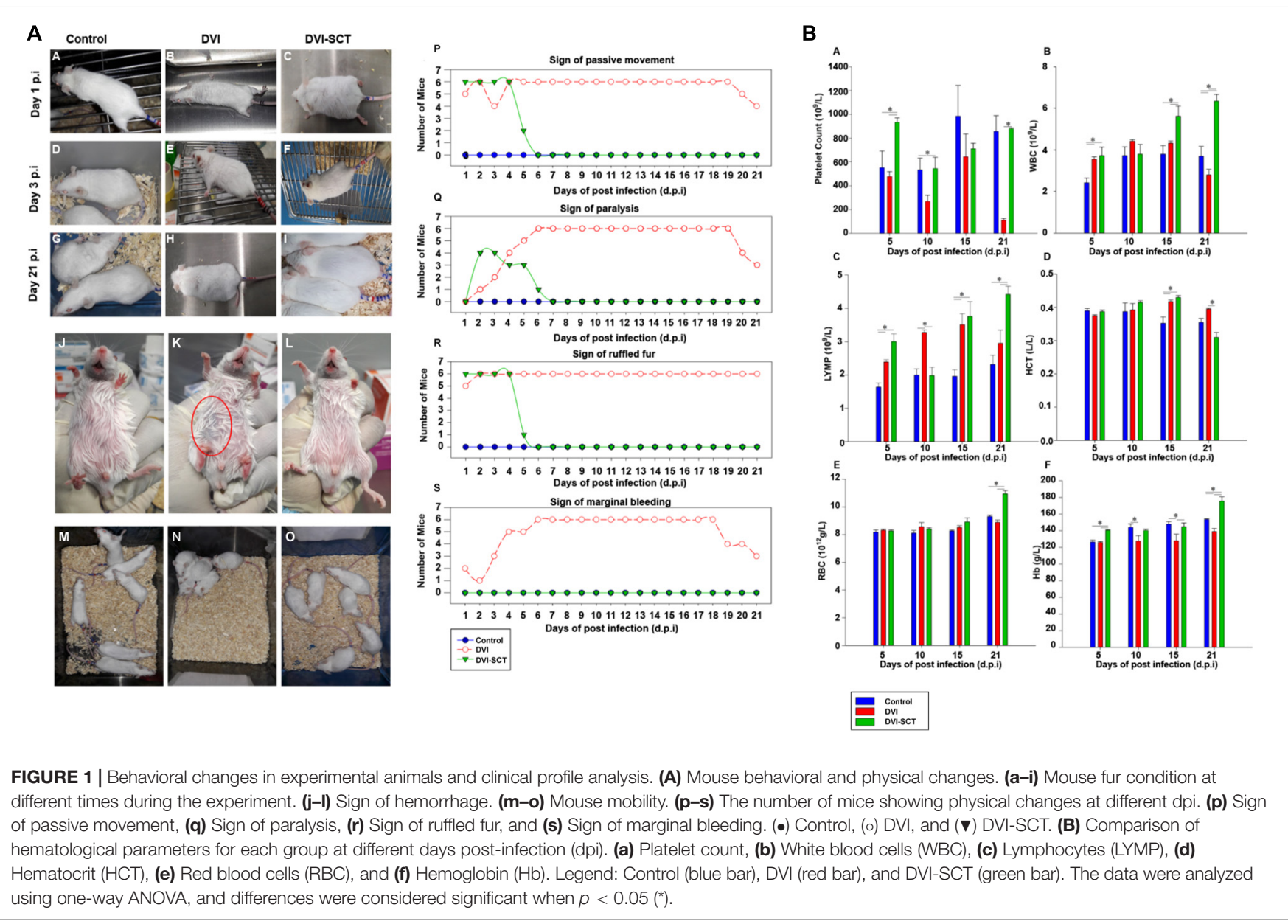

marrow to produce platelets (Woolthuis and Park, 2016). The administration of growth factor thrombopoietin (TPO) in the treatment also improved platelet production in the DVI-SCT group. In addition, including EPCs in the treatment regimen allows reconstitution of the bone marrow (BM) niche and recovery of hematopoiesis, thus increasing the platelet count (Salter et al., 2009). In addition, the white blood cell (WBC) counts observed in the DVI group in this study showed a slight increase in the initial phase but began to decline after the 15th dpi, which may be related to the BM inhibition effect of DENV similar to the changes reported in a previous dengue infection study (Tan et al., 2011; Pascutti et al., 2016) and the leukopenia state reached at the end of the experiment. In contrast, the WBC count in the DVI-SCT group remained at the same level as the control group at the initial stage and increased significantly on the 15th and 21 st dpi compared with those in the DVI group (Figure 1Bb). This indicated that the treatment with HSCs effectively increased and sustained blood cell production, including proliferation and differentiation into WBC.

Similar to changes in WBC counts, lymphocyte counts in the DVI group also increased up to 15th dpi, then began to decrease. However, in the DVI-SCT group, a high lymphocyte count was found as early as the fifth dpi, promoting a faster immune response compared to the DVI group. The lymphocyte count in the DVI-SCT group reached normal levels by the 10th dpi and continued to rise (Figure $1 \mathbf{B c}$ ). These results showed that the stem cell therapy can facilitate increased DENV clearance.

No significant difference in the hematocrit (HCT) was observed in the DVI and DVI-SCT groups, except for on the 21 st dpi, at which time the DVI group exhibited the highest HCT among all groups. This might be due to dengue-induced vascular leakage, which caused large amount of plasma exudate and increased HCT (Figures 1B,d). High HCT was shown to result in increased blood viscosity and even arterial thrombosis (Salazar Vázquez et al., 2008). Thus, the decreased HCT in the DVI-SCT group by 21 st dpi indicated that the stem cell therapy in this study is safe and effective. The platelet, WBC, and HCT data obtained from this study are comparable with several previous studies despite using different treatment materials, DENV strain, titer, and environment (Sreekanth et al., 2014, 2016).

The hemoglobin level and red blood cell (RBC) count in the DVI group were found to be low, whereas their levels in the DVISCT group consistently increased from the fifth dpi onward. The $\mathrm{RBC}$ level is similar between the groups. However, the RBC and $\mathrm{Hb}$ levels in the DVI-SCT group was noticeably increased from the 15th dpi (Figures 1Be,f).

The changes in all blood cells found in this study suggests that HSCs treatment repopulate and regenerate production of blood 
cells. HSCs $\mathrm{CD}_{3} 4^{+}$cells are progenitors that have the potential for cell renewal and myeloid and lymphoid differentiation, with their ability to home inflammation and tissue injury sites and aid in its repair (Nagy et al., 2005). In a cell-tracking study, Brudecki et al. (2012) showed that HSPCs were detected in bone marrow after 2 days of transplantation and homed to the peritoneum at a later time around the fifth day. In another study, the donorderived granulocytes and monocytes were detected after 6 days of HSPCs transplantation into lethally irradiated mice (Akashi et al., 2000). In this study, increased blood cells were detected approximately 8 days after stem cells treatment, suggesting that time is likely required for the transferred HSCs to differentiate into mature immune and other blood cells and to also to mount the protective response in the mice.

\section{Liver Biochemical Analysis}

Aspartate aminotransaminase (AST) and alanine aminotransaminase (ALT) are crucial markers in detecting liver damage, with ALT being the most prominent indicator. In this study, the AST level was significantly increased in the DVI group on the 10th and 21st dpi. In contrast, it is worth noting that in the DVI-SCT group, the AST level was maintained near to that in the control group throughout the experiment (Figure 2Ab). Meanwhile, the ALT level was highest in the DVI group except on the 15th dpi, with an increase from 30 to 50 compared to that in the control group on the 21st dpi. A pilot study by Kuo et al. (1992) showed that AST and ALT level were altered at 82.2 and $93.3 \%$ in a total case of 270 dengue fever, while another study revealed 50 cases with higher ALT level in DHF patient. However, the symptomatic dengue-infected patients showing hepatic enzyme abnormalities are able to recover faster (Seneviratne et al., 2006). Animal model studies found that the peak for both AST and ALT levels is identified, which is similar to human cases and decreased after 2 weeks (Kuo et al., 1992; Franca et al., 2010). Similar to other human and animal study, we also observed increased and decreased ALT level at 15th dpi. However, failure in recovery suggest the rise in ALT and AST levels again on the 21st dpi. Conversely, in the DVI-SCT group, the ALT level was successfully maintained with the control (Figure 2Aa). In our findings, an AST/ALT ratio indicating liver injury was obviously detected in the DVI group from 10th dpi onward. A slight increase in liver injury was observed in the DVI-SCT group on the 10th dpi, but the liver condition was maintained at par with that in the control from the 15th dpi onward (Figure 2Ac), indicating that DENV-induced liver damage has recovered after stem cell therapy.

\section{Microscopic Analysis of Liver Tissue}

Liver manifestations are not just related to viral toxicity but are also due to the immune reaction against the virus, often damaging an individual's own tissues in response to intracellular viral particles (Samanta and Sharma, 2015). Livers of control mice injected with Eagle's minimum essential medium (EMEM) and phosphate buffered saline (PBS) did not display any apoptosis and structural alteration (Figure 2Ba,d,g,j) on the 5th (Figure 2Ca), 10th (Figure 2Cd), 15th (Figure 2Cg), or 21st dpi (Figure 2Cj).
In the DVI group, the hepatic injury was observed in all the DVI mice, and the histopathological examination showed generalized steatosis (i.e., lipid accumulation within hepatocytes), tumefaction, mild apoptosis, and inflammatory cell infiltration on the fifth dpi (Figure 2Cb). These injuries were initially mild but become severe at the 10th dpi (Figures $2 \mathrm{Be}, 2 \mathrm{Ce}$ ). At 10th $\mathrm{dpi}$, the apoptosis was also increasing, characterized by a round or oval mass having an intense eosinophilic cytoplasm and dense nuclear chromatin fragments. On the 15th dpi, the number of apoptotic hepatocytes was reduced, but the signs of steatosis were predominant, with increases in hepatocyte diameters (Figures 2Ch,2Bh). Furthermore, hepatic injury was more obvious on the 21st dpi (Figure 2Ck), with significantly higher hepatocyte diameters than those observed in the control and DVI-SCT groups. Other changes include increased hepatocyte swelling, blood vessel dissection, inflammatory cell infiltration near the blood vessels, diffuse steatosis, and mild hemorrhage (Figure 2Ck). The findings can be correlated with cytokinemediated defense against the viral-infected or target cells. These immune reactions are primarily mediated by $\mathrm{T}$ cells and related interacting cells that secrete antibodies against ECs, finally resulting in the cytokine "Tsunami" acting on dengue target cells (Samanta and Sharma, 2015). These findings were comparable to human DV cases (Lawn et al., 2003; Basilio-de-Oliveira et al., 2005) and several animal models experiments (Barth et al., 2006).

In the DVI-SCT group, the liver cells also displayed diffuse steatosis, hepatocyte swelling, tumefaction, increased hepatocyte diameter, inflammatory cell infiltration, and rare blood vessel dissection with no hemorrhagic signs (Figure 2Cc) on the 5th dpi. On the 10th dpi, less inflammatory cell infiltration and no hepatocyte swelling were recorded (Figure 2Cf). Additionally, recovered tumefaction, mild steatosis, and a reduction in apoptotic cell number were seen in the DVI-SCT group (Figure 2Cf) compared with the DVI group. All signs were reduced in the DVI-SCT group by the 15th dpi, and subsequently, no inflammatory cell infiltration, hepatocyte swelling, or bleeding was observed, along with mild steatosis (Figure 2Ci). The binucleated cell percentage was consistently maintained at a value similar to that in the control until the 21st dpi (Figure 2Cl). In addition, recovered tumefaction, mild steatosis, and a reduction in apoptotic cell number were on the 10th dpi (Figure 2Bf). From the 15th dpi, no inflammatory cell infiltration, hepatocyte swelling, or bleeding was observed, along with less steatosis (Figure 2Bi). The binucleated cell percentage was consistently maintained at a value similar to that in the control until the 21st dpi (Figure 2Bl).

The stem cells injection induces liver tissue regeneration and repopulation by paracrine action of the injected cells (Lozito et al., 2009). Several growth factors and cytokines released during liver injury stimulate the migration of bone marrow cells (HSCs) to the injury site through circulation and hepatogenic differentiation and populate the liver after intravenous transplantation (Lagasse et al., 2000; Zhang et al., 2003; Ruhnke et al., 2005). Apart from that, EPCs from the bone marrow also reside at the sinusoidal endothelium (Kallis et al., 2007). Several animal studies have demonstrated that EPCs from the bone marrow have been incorporated into the sinusoidal endothelium and further 


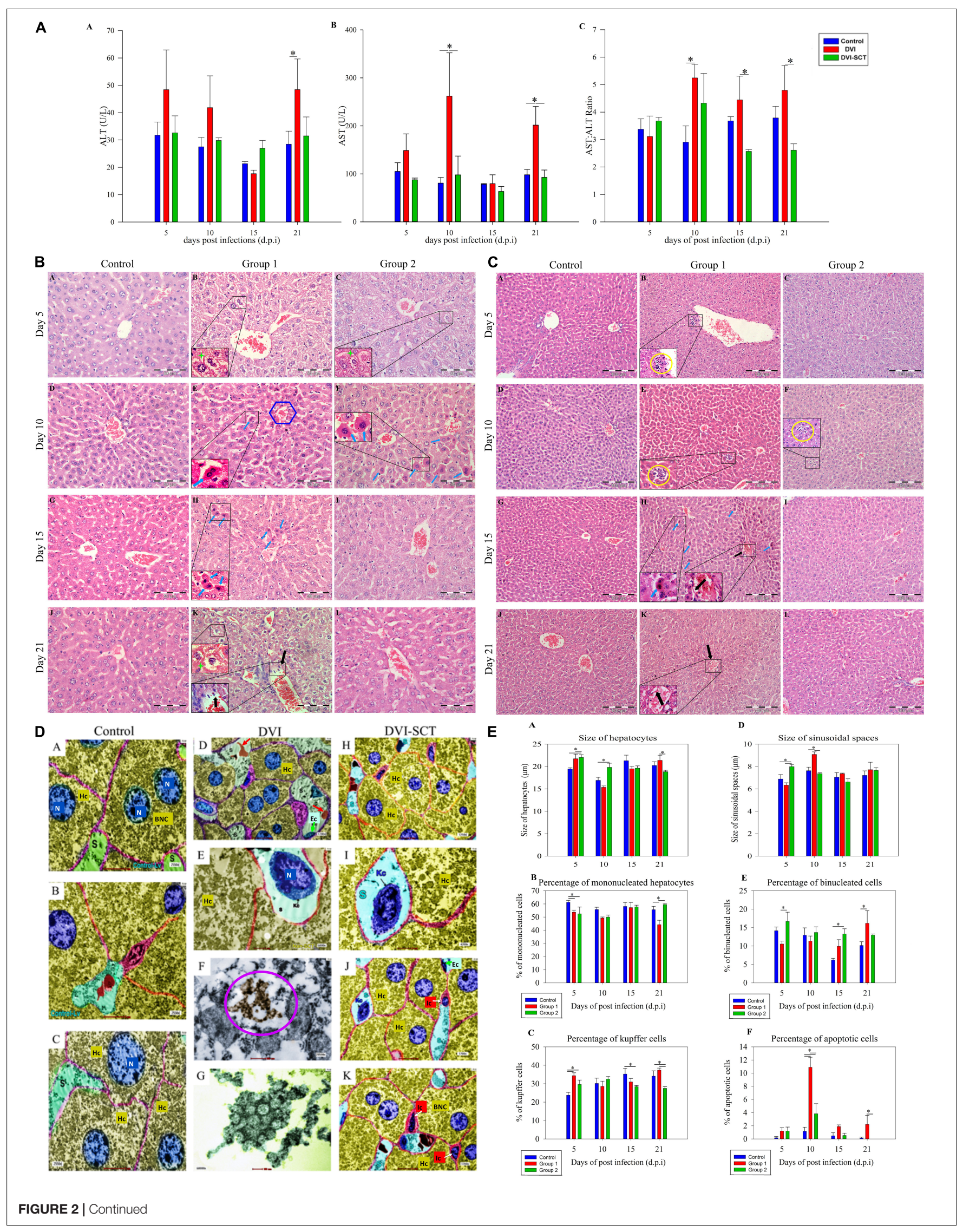


FIGURE 2 | Hepatological analysis. (A) Liver biochemical analysis (ALT, AST) at different dpi in different groups. Legend: Control (blue bar), DVI (red bar), DVI-SCT (green bar). (B) Comparison of histological examination of liver at different dpi in different groups ( $\times 400$ magnification). Legend: Steatosis cells (green star), inflammatory cell infiltration (yellow round), apoptotic cells (blue arrow) and blood vessel tear (black arrow), and central vein (blue hexagon). (C) Liver microscopic images at different dpi in different groups (× 200 magnification). Legend: apoptotic cells (blue arrow) and blood vessel tear (black arrow). (D) Liver transmission electron microscopy images of DENV2-infected mice. N, nucleus; BNC, binucleated cells; MNC, mononucleated cells; Ec, endothelial cells; Hc, hepatocytes; Kc, Kupffer cells; S, sinusoids; Ic, Ito cell; red arrow, red blood cells; purple circle, DENV particles. (E) Cell population counts at different dpi. Legend: Control (blue bar), DVI (red bar), and DVI-SCT (green bar). The data were analyzed using one-way ANOVA, and differences were considered significant when $p<0.05$ ( ${ }^{*}$.

applied for the reconstruction of hepatic sinusoids during liver regeneration with the elevated level of hepatocyte growth factor (HGF) and vascular endothelial growth factor (VEGF) (Fujii et al., 2002; Taniguchi et al., 2006) and hence overall repaired the injured liver after dengue infection.

\section{Transmission Electron Microscopy of Liver Tissue}

The transmission electron microscopy (TEM) observations of the liver tissue detailed the presence of mononucleated and binucleated cells and the variations in the size of the nucleus in the hepatocytes (Figure 2D). Compared with the control group, the DVI group showed complex viral-induced subcellular changes. The hepatocytes exhibited mononucleated cells (MNC), binucleated (BNC), Kupffer cells, and Ito cells, indicating both inflammatory response and the reparative responses (Figures 2Dd,e). Furthermore, the sinusoidal spaces were more frequently noted to contain Kupffer cells in the DVI group than in the DVI-SCT group. This also demonstrates the increased immune reaction in the DVI-SCT group due to the dominating actions of the virus. The DVI-SCT group exhibited a relatively larger cell size at the same magnification than the control and DVI groups, which indicates tumefaction (Figures 2Dh,j,k). Virus-like particles were observed inside hepatocytes in the DVI group (Figures 2Df,g) but not in the hepatocytes in DVI-SCT group after careful evaluation of the sectioned area. These results provide evidence of the relative clearance of the virus in the DVISCT group.

\section{Liver Cell Population Count}

Livers of control mice showed a significant increase in hepatocyte diameter alongside diminished sinusoidal space (Figures 2Ea,b). Predominantly mononucleated hepatocytes with less binucleated hepatocytes and an increased number of Kupffer cells were also demonstrated in comparison with the control group (Figures 2Ed,e,f) $(p>0.05)$. On the 10th dpi, reduced hepatocyte diameter and extended sinusoidal space were observed along with the presence of diffuse hepatocyte apoptosis near the central vein (Figures 2Eb,c).

On the 21st dpi, the percentage of binucleated hepatocytes and Kupffer cells was significantly higher in the DVI group than in the control and DVI-SCT groups (Figures 2Ee,f). In the DVISCT group, the percentage of binucleated cells was at its highest on the fifth dpi (Figure 2Ee), alongside wider sinusoidal spaces (Figure 2Eb) and a lower percentage of Kupffer cells (Figure 2Ef), compared with the DVI group. In the DVI-SCT group at the 15th dpi, quantification of the apoptotic cells yielded a lower percentage compared with that in the DVI group, and the number consistently remained near that of the control level until the 21st dpi (Figure 2Ec). Furthermore, the percentage of Kupffer cells was significantly lower than that in the control group and only slightly lower than that in the DVI group (Figure 2Ef). In addition, the size of the hepatocytes and the percentage of mononucleated hepatocytes in the DVI-SCT group was not significantly different than those in the control and DVI groups on the 15th dpi. Nevertheless, the DVI-SCT group exhibited reduced liver injury by the $21 \mathrm{st} \mathrm{dpi}$, as well as reduced hepatocyte size (characterized by swelling and rounding up of the hepatocyte which leads loss of hepatocyte shape), a significantly higher mononucleated hepatocyte percentage, and a lower percentage of Kupffer cells (Figures 2Ea,d,f).

The low number of binucleated hepatocytes in the DVI group at early stage indicated that liver recovery phase occurs only after 21st dpi. This may be one of the reasons for the mortality of dengue due to liver failure. In contrast, the number of binuclear cells in the DVI-SCT group achieved its peak on the fifth dpi and thereafter remained at a level higher than in the control group, suggesting that the liver repair has begun in the early stages of infection (Woolthuis and Park, 2016). In addition, the important role of Kupffer cells in infection and inflammation explains their high levels in the DVI group, suggesting that viral activity persisted until the end of the experiment (Paes et al., 2009). In contrast, the DVI-SCT group showed a lower percentage of Kupffer cells throughout the experiment. The results supported that the combined stem cell therapy contributes to liver regeneration and recovery during DENV infection.

\section{Immunohistochemical Study of Liver Tissue}

To assess the ability of the stem cell cocktail infusion in eliminating DENV from dengue-infected mice, immunohistochemical staining was performed to detect the presence of viral antigen in livers of DVI and DVI-SCT mice. Immunohistochemistry showed that mouse hepatocytes positive for DENV antigens (green fluorescence) were more prominent in the DVI group than in the DVI-SCT group throughout the experiment (Figure 3A). Despite the slight reduction in DENV antigens observed in the DVI group by the 21 st dpi, the virus was not completely cleared from the liver cell population. In contrast, the DVI-SCT group displayed minimal expression of DENV antigens, suggesting that the virus was eliminated from the liver cells by the 21 st dpi. Here, we have provided a qualitative data on the presence of virus in infected mice. It is also very important to provide quantitative data to have a stronger evidence of viral clearance. Thus, dengue virus 


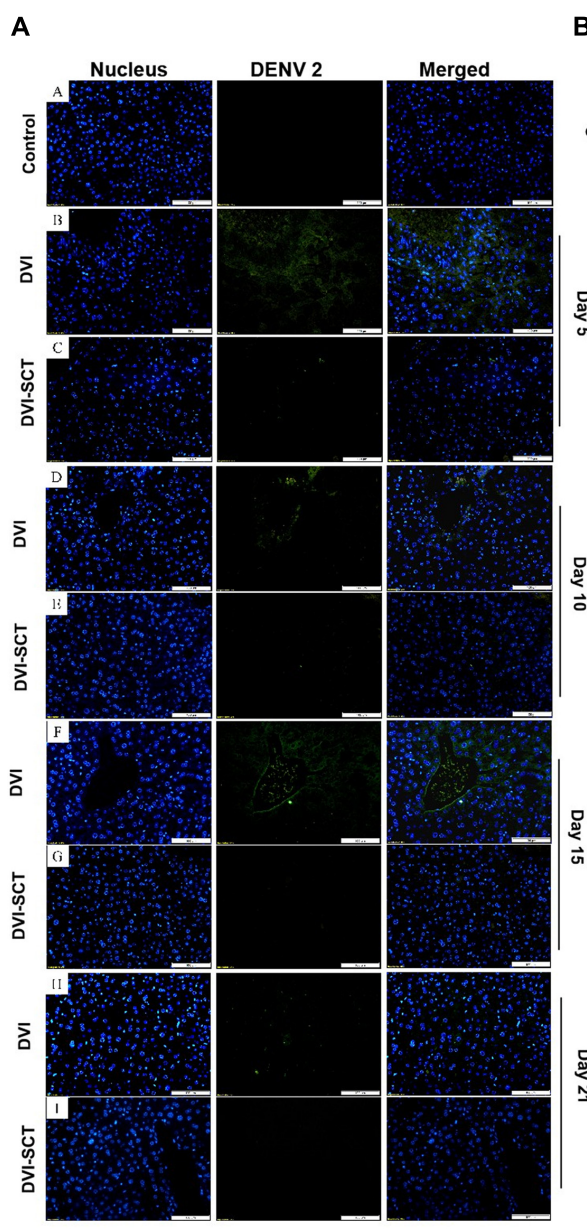

B

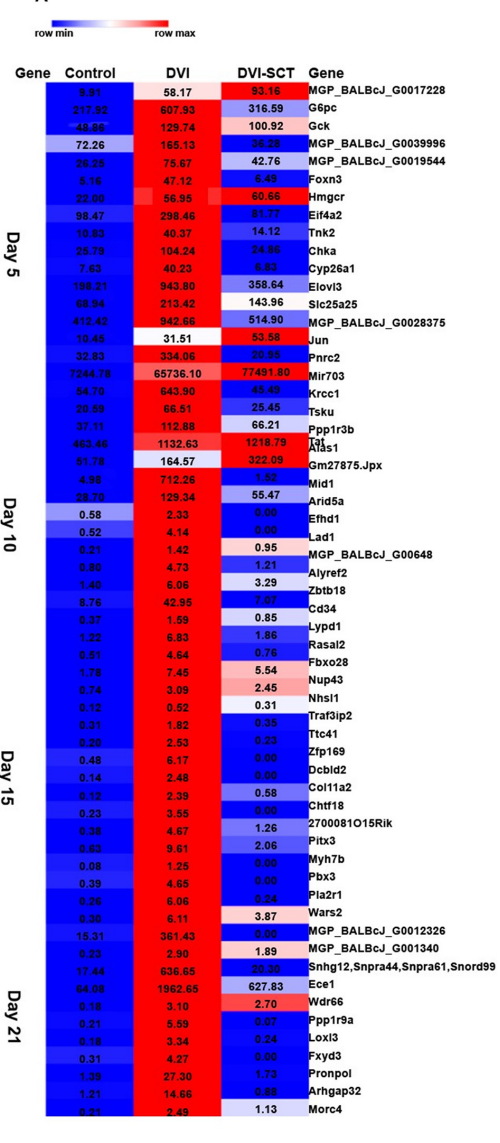

B

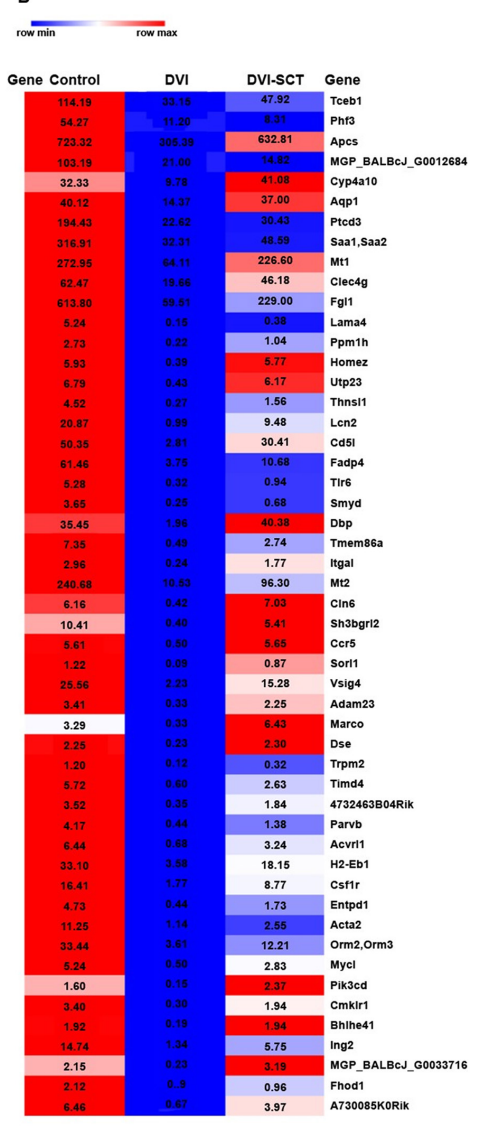

C
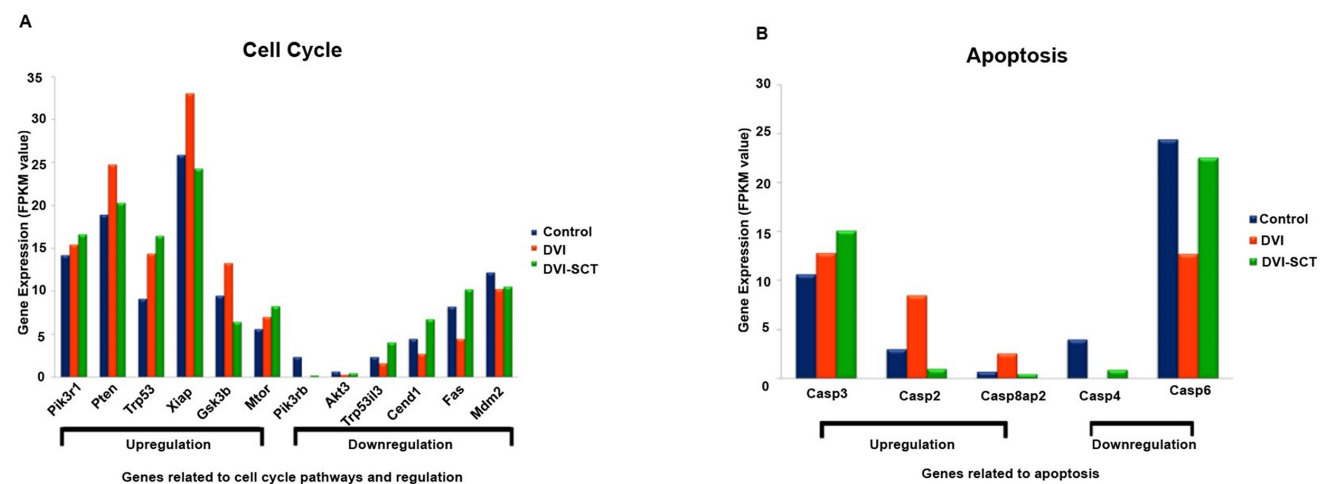

FIGURE 3 | Immunohistochemical assessment and next-generation sequencing. (A) Immunohistochemical study of DENV-infected BALB/c mice liver at different dpi. Liver sections were captured at a magnification of $\times 200$ under a fluorescence microscope. (a) Control group, (b,c) liver sections of DENV-infected and stem-cells-treated mice on the 5th dpi, (d,e) 10th dpi, (f,g) 15th dpi, and $\mathbf{( h , i )} 21$ st dpi. Blue (DAPI-stained nuclei) and green (Alexa Fluor stained DENV antigen). The green fluorescence seen in the DVI group was diminished in the DVI-SCT group. (B) Heatmap of upregulated genes. (C) Heatmap of downregulated genes. The dysregulation (up - or downregulation) of liver genes was quantified in three sets of BALB/c mice: control mice injected with Eagle's minimum essential medium (EMEM) and phosphate-buffered saline (PBS); DVI mice infected with DENV2; and DVI-SCT mice infected with DENV2 and treated with HSCs, EPCs, and growth factor $(n=3)$. The heat maps generated were quantified based on the row minimum and row maximum values (specific for each gene), with red indicating genes highly upregulated and blue depicting genes downregulated. (a) Gene expression related to cell cycle pathways and regulation. The genes Pik3r1, Pten, Trp53, Xiap, Gsk3b, and Mtor showed gene expression upregulation following dengue virus infection (DVI), which were either corrected to reflect the control levels (Pten, Xiap, and Gsk3b) or continuously upregulated to benefit mice (Pik3r1, Trp53, and Mtor) upon stem cell treatment (DVI-SCT). Meanwhile, the genes Pik3r6, Akt3, Trp53i13, Ccnd1, Fas, and Mdm2 showed downregulation upon dengue virus infection (DVI), which was also all corrected to reflect control values upon stem cell treatment (DVI-SCT). (b) Upregulated and downregulated genes related to apoptosis. The genes Casp3, Casp2, and Casp8ap2 showed upregulated gene expression following dengue virus infection (DVI) and was corrected to reflect control levels (Casp2 and Casp8ap2) or continuously upregulated to benefit mice (Casp3) upon stem cell treatment (DVI-SCT). Meanwhile, the genes Casp4 and Casp6 showed downregulation upon dengue virus infection (DVI), which was corrected to reflect control levels upon stem cell treatment (DVI-SCT). 
screening assays, such as immunofluorescence assay, IC50, FFU assay, quantitative reverse transcription PCR (RT-PCR) assay, and plaque assay method, are ample and fit for the high-throughput screening of virus clearance (Low et al., 2011; Sreekanth et al., 2019).

HSCs are urged by the signals of innate and adaptive immunity in order to respond to the pathogen-specific infection through systematic cytokine stimulation. HSCs express various signaling receptors, which instantly engaged in the infection responses, by its attachment with the infectious ligands and cytokines such as toll-like receptors (TLRs), tumor necrosis factor alpha (TNF) receptor, and interferon (IFN) receptors (Baldridge et al., 2010). The infection signal produced by DENV activates the HSCs and surge the differentiation of HSCs. This differentiation generates the immune effector cells and eventually neutralizes the early infections (Baldridge et al., 2011). HSC also modifies the cytokine secretion profile of T-naive cells, NK cells, effectors, and dendritic cells to induce a tolerant phenotype, secretion of more proinflammatory and anti-inflammatory cytokines, TNF- $\alpha$, and decreased IFN- $\gamma$, with suppressively stimulated IL-4 and IL-10 (Lozito et al., 2008). Some evidence suggests that HSCs act as anti-inflammatory and immunoregulatory (Lozito et al., 2008). A recent study demonstrated treatment for COVID-19 infection using HSCs. The study suggested that the mechanism of HSC to eliminate viruses is by reducing inflammatory cytokines and by increasing anti-inflammatory cytokines that make the virus inactive (Frias-Staheli et al., 2014; Purwati Sumorejo et al., 2020).

Moreover, HSCs also reconstitute and differentiate into immune cell populations in the liver (Jiang et al., 2013), which initiate the phagocytosis of virus and its clearance from the cell. Previous reports have demonstrated the direct dialogue between HSCs outside niches and the immune system through "in" signaling and "out" signaling. The DENV as an "in" signal stimulates the differentiation of HSCs into immune cells (Zheng et al., 2011), which may explain the mechanism of viral clearance seen in the DVI-SCT group in this study. Our findings further validated the ability of the transplanted stem cells to eliminate DENV from the host cells. Although the virus is not visible after stem cells treatment, the mechanism at which stage of the viral replication does the treatment combats is yet to be clarified.

\section{Gene Expression Profiling Using MiSeq Next-Generation Sequencing}

To further analyze the molecular level changes induced by DENV infection and stem cell treatment in dengue-infected mice, RNA sequencing (RNA-seq) using MiSeq next-generation sequencing was employed in this study. Transcriptomics analysis comparing gene expression patterns in different experimental groups showed that of the 38,167 genes expressed in the control group (total fragments per kilobase of exon per million mapped reads, FPKM $=0$ ) and the DVI group (FPKM > 10), 15 were novel genes expressed exclusively in DVI samples, thus suggesting that these 15 genes are triggered by dengue infection (Table 1). These novel genes maybe one of the reasons for the cell dysfunction during dengue infection. Similarly, the expression of novel genes between the DVI and DVI-SCT groups were cross-checked to

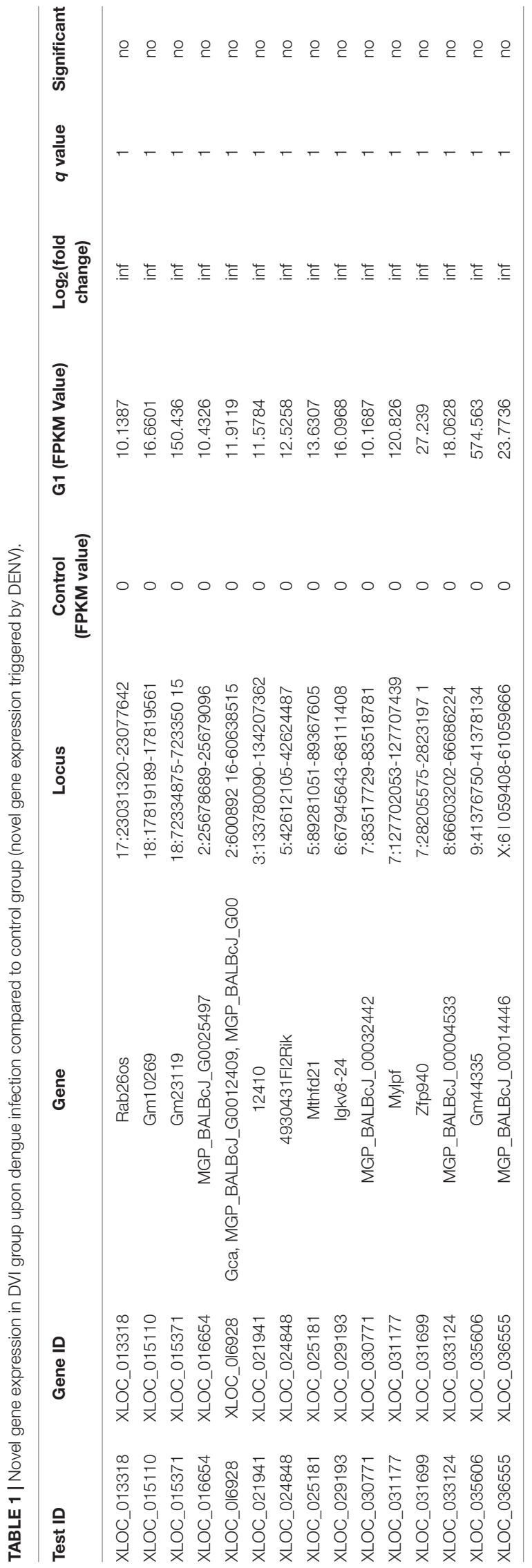


elucidate the presence of novel genes triggered exclusively by the stem cell treatment. Of the total 38,166 genes expressed, 38 newly expressed genes were identified between the control $(\mathrm{FPKM}=0)$ and DVI-SCT $($ FPKM > 10) groups (Table 2). These novel genes triggered by the stem cell treatment may be responsible for DENV clearance and for reversing the effects of DENV infection. The occurrence of novel gene expression triggered by either DENV2 infection or stem cell treatments can serve as a vital foundation for future studies. Research on these newly expressed genes may aid in elucidating the interactions between DENV and host or the role of stem cells in DENV clearance.

Thereafter, the changes in gene expression profiles between groups were calculated by the Cuffdiff method. The genetic profiles demonstrated both upregulation and downregulation of certain liver-associated genes in mice of all three groups. After analysis, it was found that 59 genes were significantly upregulated in the DVI group, and in the DVI-SCT group, 47 of the genes were successfully corrected similar to that of control group, while the remaining genes showed downregulation (Figure 3Ba and Supplementary Table 2). Figure 3Ba shows 59 significantly upregulated genes following DENV infection, among which 47 genes (e.g., G6pc, MGP_BALBcJ_G0039996, MGP_BALBcJ_G0019544, Foxn3, and Eif4a2) were successfully corrected to preinfection (control) levels, while the rest (e.g., MGP_BALBcJ_G0017228, Gck, Hmgcr, and Jun) showed insignificant levels of upregulation. On the other hand, 51 genes were significantly downregulated in the DVI group, and 30 of the genes were successfully corrected to the control level in the DVISCT group, while the remaining genes showed a downregulation level (Figure 3Bb and Supplementary Table 1). Figure 3Bb shows 51 significantly downregulated genes following DENV infection, among which 30 genes (e.g., Tceb1, Phf3, and Ptcd) were successfully corrected to preinfection (control) levels, while the remaining genes (e.g., Apcs, Cyp4a10, and Aqp1) did not show evidence of normalization. The normalization in the expression levels of the DENV-induced dysregulated genes in the DVISCT group showed that stem cells are able to modulate gene dysregulation caused by dengue infection. Stem cells treatment corrected up- or downregulated genes in the infection fully (or partially) to the normalized gene level, suggesting the potential of stem cells in treating DENV infection at the genetic level.

To determine the function of a particular differentially expressed gene, all differentially expressed genes were mapped into a Gene Ontology (GO) database. Twelve genes involved in the cell cycle pathway and regulation were selected for analysis (Figure 3Ca). Of the 12 selected genes, 6 showed upregulation, while the other 6 showed downregulation upon DENV2 infection. All the gene (both up- and downregulated) expression levels were normalized following treatment with stem cells except for three of the upregulated genes. Upregulation of the gene Gsk3b, which is involved in metabolic pathways, including inflammation, stress, mitochondrial dysfunction, and apoptosis (Jope et al., 2007), was adjusted to reflect control levels. The phosphatidylinositol-3-kinase/protein kinase B (PI3K-Akt) pathway gene subunit, $P i k 3 r 1$, which plays a key role in cell cycle, apoptosis, and DNA repair (Franke, 2008), was continuously upregulated upon stem cell treatment to ensure increased platelet proliferation and white blood cell count, countering the apoptosis and thrombocytopenia seen in dengue infection. This cements the hypothesis that both HSCs and EPCs play a vital role in ensuring regulation of cell cycle pathways and counteracting the negative effects of dengue infection.

In addition, genes related to apoptosis were also analyzed in the obtained NGS data (Figure $\mathbf{3 C b}$ ). The results showed that the all the genes related to apoptotic pathways (Casp2, 3, 4, and 6 and 8ap2) that were dysregulated in dengue infection were corrected to a level similar to that in control samples following stem cell treatment. This shows that the widespread apoptosis caused by dengue infection was reduced and regulated by stem cell treatment, ensuring correction of gene expression and a decrease in swelling and the size of hepatocytes in addition to reduced apoptosis and sinusoidal spaces in hepatocytes (Paes et al., 2005; Sakinah et al., 2017). Taken together, the NGS data further validated the histopathological findings of this study, thus suggesting that stem cell treatment is a superior treatment option for dengue infection.

In the recent years, the incidence of dengue has continued to increase, but there is currently no rapid and effective treatment available. This study demonstrates stem cell therapy as a promising management to reduce the global burden of dengue. Since this is the first study done to treat dengue infection with stem cells, we faced several limitations such as very little research is written on this subject to use as a reference and inadequate blood samples to perform additional quantitative test for validation. Hence, this study could be an exploratory study that lay the groundwork for more complete research in the future. Our study recommends a more detailed study on host responses, mainly the interferon (IFN) signaling to stem cell therapy, which would provide the mechanism underlying the host-pathogen interaction A robust study is required to provide more evidence for the effectiveness of this treatment including observing the effect of the treatment in other organs such as the spleen, kidney, and brain, identifying the virus load and gamma-GGT levels and elucidating the antiviral genes in tissues and serums, exploring the presence of antibodies and antigens of the virus by RT-PCR, characterizing various proinflammatory cytokines that are involved in dengue infection, and using TUNEL assay to analyze apoptosis.

\section{MATERIALS AND METHODS}

\section{Mouse Model of Dengue Infection and Stem Cell Therapy}

This study was carried out in the animal house, Faculty of Veterinary Medicine, UPM. All in vivo procedures were performed with the ethical approval of the IACUC, Universiti Putra Malaysia (ref. no. UPM/IACUC/AUP-R017/2015). Seventy-two 8-week-old male BALB/c mice (20-25 g) were acclimated to the environment for 1 week and divided into three groups: control, DVI, and DVI-SCT groups (24 mice each group). EMEM (control group) and DENV 2 $\left(1.25 \times 10^{5} \mathrm{FFU} / \mathrm{ml}\right)(\mathrm{DVI}$ and DVI-SCT groups) were injected intraperitoneally according to different groups for 2 consecutive 
TABLE 2 | Novel gene expression in DVI-SCT group compared to DVI group (novel gene expression triggered by stem cell treatment).

\begin{tabular}{|c|c|c|c|c|c|c|c|c|}
\hline Test ID & Gene ID & Gene & Locus & $\begin{array}{c}\text { Control } \\
\text { (FPKM value) }\end{array}$ & G2 (FPKM value) & $\begin{array}{c}\log _{2} \text { (fold } \\
\text { change) }\end{array}$ & $q$ value & Significant \\
\hline XLOC_003186 & XLOC_003186 & Sult3a 1 & 10:31283423-3 1298517 & 0 & 48.8338 & $\inf$ & 0.007606 & yes \\
\hline XLOC_021585 & XLOC_021585 & Hao2 & 3:96548755-96566667 & 0 & 36.539 & $\inf$ & 0.007606 & yes \\
\hline XLOC_021924 & XLOC_021924 & MGP_BALBcJ_00006282 & 3:131637308-13 1643455 & 0 & 41.0333 & $\inf$ & 0.007606 & yes \\
\hline XLOC_30257 & XLOC_30257 & MGP_BALBcJ_G0040533 & 7:24101650-24108542 & 0 & 52.1268 & $\inf$ & 0.007606 & yes \\
\hline XLOC_30258 & XLOC_30258 & Сур2b9 & 7:24185787-24223302 & 0 & 167.403 & $\inf$ & 0.007606 & yes \\
\hline XLOC_030259 & XLOC_030259 & MGP_BALB cJ_G0031867 & 7:24292602-2433421 1 & 0 & 209.279 & $\inf$ & 0.007606 & yes \\
\hline XLOC_031499 & XLOC_031499 & Sult2a3 & 7:11494777 -11551540 & 0 & 34.5995 & $\inf$ & 0.007606 & Yes \\
\hline XLOC_000447 & XLOC_000447 & Gm27512 & 1:61169239-61202084 & 0 & 436.136 & $\inf$ & 1 & no \\
\hline XLOC_000503 & XLOC_000503 & Gm25360 & 1:70252534-70252725 & 0 & 94.1518 & $\inf$ & 1 & no \\
\hline XLOC_001981 & XLOC_001981 & MGP_BALBcJ_G0010132 & 1:58438442-58523586 & 0 & 33.3261 & $\inf$ & 1 & no \\
\hline XLOC_002729 & XLOC_002729 & Fmo3 & |:161974097-162100359 & 0 & 110.528 & $\inf$ & 0.338451 & no \\
\hline XLOC_003112 & XLOC_003112 & MGP_BALBcJ_G0036352 & 10:15144200-15155939 & 0 & 29.8837 & $\inf$ & 1 & no \\
\hline XLOC_003209 & XLOC_003209 & Rnu3a & | 0:37833676-37833890 & 0 & 116.346 & $\inf$ & 1 & no \\
\hline XLOC_005084 & XLOC_005084 & Gml7305 & 11:67460712-67487429 & 0 & 10.2884 & $\inf$ & 1 & no \\
\hline XLOC_005422 & XLOC_005422 & MGP_BALBCJ_G0018893 & 11:92952527-92956552 & 0 & 10.5147 & $\inf$ & 1 & no \\
\hline XLOC_007533 & XLOC_007533 & Acot3 & 12:81841321-81863070 & 0 & 30.5984 & $\inf$ & 0.478207 & no \\
\hline XLOC_007547 & XLOC_007547 & Gm 17139 & 12:82747202-82820080 & 0 & 14.7918 & $\inf$ & 1 & no \\
\hline XLOC_012625 & XLOC_012625 & Gml 0076 & 16:468038-468405 & 0 & 14.5565 & inf & 1 & no \\
\hline XLOC_013281 & XLOC_013281 & Gml0226 & 17:19869811-19870066 & 0 & 10.0665 & $\inf$ & 1 & no \\
\hline XLOC_014038 & XLOC_014038 & Snora78 & 17:23277562-2327 7996 & 0 & 377.441 & $\inf$ & 1 & no \\
\hline XLOC_014192 & XLOC_014192 & MGP_BALBcJ_G0003360 & 17:32930035-353 87947 & 0 & 17.1602 & $\inf$ & । & no \\
\hline XLOC_016013 & XLOC_016013 & Slc22a26 & 19:4653942-4675569 & 0 & 19.7565 & $\inf$ & । & no \\
\hline XLOC_016549 & XLOC_016549 & mt-Tsl & 2:19329205-19618562 & 0 & 288021 & $\inf$ & । & no \\
\hline XLOC_017204 & XLOC_017204 & MGP_BALBCJ_G00364 14 & 2:88663442-88687150 & 0 & 11.3819 & $\inf$ & । & no \\
\hline XLOC 017433 & XLOC 017433 & MGP_BALBCJ_G0011813 & 2:119439014-119486495 & 0 & 10.9909 & $\inf$ & 1 & no \\
\hline XLOC_018260 & XLOC_018260 & MGP_BALBcJ_G0036413 & 2:26820995-26941552 & 0 & 19.9283 & $\inf$ & । & no \\
\hline XLOC_020938 & XLOC_020938 & Gm33051 & 3:25629719-25640724 & 0 & 37.8406 & $\inf$ & 1 & no \\
\hline XLOC_022066 & XLOC_022066 & Gm24494 & 3:151934096-151939819 & 0 & 52395.6 & $\inf$ & 1 & no \\
\hline XLOC_023192 & XLOC_023192 & Gm I3203 & 4: 146653969-146656880 & 0 & 10.6962 & $\inf$ & 1 & no \\
\hline XLOC_024169 & XLOC_024169 & Gm26716 & 4: 127942608-127976912 & 0 & 12.0673 & $\inf$ & 1 & no \\
\hline XLOC_029178 & XLOC_029178 & lgkv5-39 & 6:67607309-67607863 & 0 & 11.8116 & $\inf$ & 1 & no \\
\hline XLOC_029675 & XLOC_029675 & Gm44096 & 6:122520707-122613222 & 0 & 17.595 & $\inf$ & । & no \\
\hline XLOC_030266 & XLOC_030266 & Cyp2gl & 7:24971909-24994733 & 0 & 18.6062 & $\inf$ & 1 & no \\
\hline XLOC_031497 & XLOC_031497 & MGP_BALBcJ_G003165S,Sult2a2 & 7:11303713-11394048 & 0 & 177.379 & $\inf$ & 0.338451 & no \\
\hline XLOC_031503 & XLOC_031503 & Sult2a6 & 7: 11656322-11692614 & 0 & 153.433 & $\inf$ & 0.338451 & no \\
\hline XLOC_033861 & XLOC_033861 & Gm28063 & 8:54953411-54956948 & 0 & 14.4561 & $\inf$ & 1 & no \\
\hline XLOC_034394 & XLOC_034394 & Gm24357 & 9: 12002860-12002934 & 0 & 145893 & $\inf$ & 1 & no \\
\hline XLOC_035890 & XLOC_035890 & Rn7sk & 9:75583363-75583694 & 0 & 11.4497 & $\inf$ & 1 & no \\
\hline
\end{tabular}


days (500 $\mu \mathrm{l} /$ day). Thereafter, the control group and the DVI group were intravenously injected with PBS for 3 consecutive days. The DVI-SCT group was injected with specific stem cells [HSCs $\left(2.5 \times 10^{5}\right.$ cells per mouse $)$ and EPCs $\left(1 \times 10^{6}\right.$ cells per mouse)] and growth factors ( $1 \mu \mathrm{g} / \mathrm{kg} / \mathrm{day}$ for 3 days). After 2 weeks, PBS (control and DVI groups) and specific stem cells [HSCs $\left(2.5 \times 10^{5}\right.$ cells per mouse $)$ and EPCs $\left(1 \times 10^{6}\right.$ cells per mouse)] and growth factor $(1 \mu \mathrm{g} / \mathrm{kg} /$ day for 1 day) were intravenously injected in $100 \mu \mathrm{l}$ according to different groups. Blood of each group was collected for hematological analysis (scil Vet abc ${ }^{\mathrm{TM}}$, Horiba, Germany) on 5, 10, 15, and 21 dpi and at the above four time points; six mice from each group were sacrificed and collected for histological examination, DENV immunohistochemical staining, and differentially expressed genes and transcriptomics analysis.

\section{Propagation of DENV 2}

Aedes albopictus clone (C6/36) cells (ATCC ${ }^{\circledR}$ CRL-1660 ${ }^{\mathrm{TM}}$, United States) were grown in a T25 flask, and the cells were subcultured until an adequate number of cells were obtained for DENV 2 viral inoculation and propagation. The procedure was performed following that described by Ip and Liao (2010) with different culture medium, as suggested by ATCC [Eagle's minimum essential media (EMEM, Biowest, Riverside, CA, United States) supplemented with $10 \%$ fetal bovine serum (FBS, Biowest) and $1 \%$ penicillin-streptomycin (Biowest)] (Ip and Liao, 2010). When the C6/36 cells reached $80 \%$ confluency at passage 3,1 ml of DENV 2, a clinical isolate obtained from the virology laboratory at UPM, was inoculated into the confluent cells. The flask containing DENV 2 was incubated at $25^{\circ} \mathrm{C}$ for $1 \mathrm{~h}$ with $10 \mathrm{rpm}$ agitation. After that, $5 \mathrm{ml}$ of EMEM supplemented with 2\% FBS was added, and the flask was incubated at $28^{\circ} \mathrm{C}$ for 8 days at a multiplicity of infection (MOI) of 0.1. The cytopathic effects (CPEs) were observed every day. After 8 days of incubation, the virus was isolated using a rapid freeze-thaw technique. This procedure was repeated for up to 10 passages to obtain a higher virus titer (Mota and RicoHesse, 2011; Martinez-Gutierrez et al., 2014). For confirmation of the DENV serotype, an immunofluorescence assay was performed using antibodies directed against all four DV serotypes following a protocol reported by Medina et al. (2012). Briefly, an immunofluorescence assay was performed using cultured infected cells that were fixed on a multiwell slide (Supplementary Figure 1). The cells were stained with DV serotype-specific monoclonal antibodies (mAbs, Merck Millipore, Darmstadt, Germany) and then with fluorescein isothiocyanate (FITC)conjugated antimouse antibody (Merck Millipore). The slide was later examined under a fluorescence light microscope at $\times 100$ magnification for observation of positive cells. The culture supernatant containing DENV 2 was collected and filtered through a $0.22 \mu \mathrm{M}$ filter. A foci forming assay was also performed at passage 10 . Vero cells were inoculated with serially diluted DENV 2, followed by staining with monoclonal anti-DENV antibodies, secondary antibodies conjugated with enzymes, and finally with the metal enhanced 3,3'-diaminobenzidine (DAB) substrate. The dark brown foci were visualized and counted under a stereo microscope on the fifth dpi. The virus titer was obtained $\left(1.25 \times 10^{5} \mathrm{FFU} / \mathrm{ml}\right)$ through viral quantitation using the following formula: virus titer, FFU/ml (foci forming unit per milliliter $)=$ average of foci/dilution factor $\times$ virus inoculums volume (Zandi et al., 2012). The virus stock is stored at $-80^{\circ} \mathrm{C}$ for subsequent infection.

\section{Preparation of Hematopoietic Stem Cells}

Hematopoietic stem cells were isolated from bone marrow cells of five 8-week-old BALB/c mice. Briefly, tibias and femurs were obtained and cut open to expose the bone marrow cavity. The bone marrow cavity was carefully punctured using a 26G needle, and the bone marrow was flushed out with Dulbecco's modified Eagle's medium (DMEM, Gibco) supplemented with 2\% FBS (Gibco, Waltham, MA, United States) until there was no visible reddish line in the bone. Cells were placed into a $50 \mathrm{ml}$ tube for isolation of CD117-positive cells (HSCs). The CD117-positive cells were isolated using a CD117-positive selection kit (Easysep ${ }^{\circledR}$, STEMCELL Technologies, Singapore) with an EasySep ${ }^{\circledR}$ magnet (Kroeger et al., 2009).

Cell suspension with a concentration of $1 \times 10^{7}$ cells per $100 \mu \mathrm{l}$ was prepared in PBS (Invitrogen, Carlsbad, CA, United States) with 2\% FBS. Cells were incubated in CD117 PE labeling reagent $(50 \mu \mathrm{l} / \mathrm{ml})$ with mixing for $15 \mathrm{~min}$ at room temperature. PE selection cocktail $(70 \mu \mathrm{l} / \mathrm{ml})$ was applied for $15 \mathrm{~min}$ at room temperature. Next, the cells were mixed with $50 \mu \mathrm{l} / \mathrm{ml}$ nanoparticles and incubated at room temperature for $10 \mathrm{~min}$. The cell suspension was brought to a total volume of $2.5 \mathrm{ml}$ and set aside for $5 \mathrm{~min}$. The EasySep ${ }^{\circledR}$ magnet was inverted in one continuous motion to pour off the supernatant. This washing method was repeated four times to avoid cell contamination. Finally, the tube was removed from the magnet, and the cells were resuspended in DMEM supplemented with 15\% FBS for expansion (Dudeck et al., 2011). CD 117-positive cells were cultured in high-glucose DMEM supplemented with 15\% FBS, $1 \%$ penicillin-streptomycin (Biowest), $100 \mathrm{ng} / \mathrm{ml}$ murine stem cell factor (SCF) (BioVision, Milpitas, CA, United States), $6 \mathrm{ng} / \mathrm{ml}$ murine IL-3 (BioVision), and $10 \mathrm{ng} / \mathrm{ml}$ human IL-6 (BioVision). The medium was replaced with fresh medium every 3 days until the cells reached confluence, and cell culture was continued until the cells reached passage 3 (Sekulovic et al., 2008). The expanded cells were further characterized for HSCs surface markers [with APC-conjugated CD133, FITC-conjugated CD34, FITC-conjugated CD135, and PE-conjugated CD117 antibodies (eBioscience, San Diego, CA, United States)] using flow cytometry (FACS Aria III cell sorter) (Frascoli and Proietti, 2012).

\section{Preparation of Endothelial Progenitor Cells}

$\mathrm{BALB} / \mathrm{c}$ mouse bone marrow-derived endothelial progenitor cells were purchased from Cell Biologics, Inc. (Cell Biologics, Chicago, IL, United States). The cell revival and culture protocols were carried out according to the company instructions. The cells in the cryovial were quickly thawed in a $37^{\circ} \mathrm{C}$ water bath for $<1 \mathrm{~min}$, resuspended in $7 \mathrm{ml}$ of prewarmed Cell Biologics cell culture medium, and added to a fresh tube. After centrifugation, 
the cells were resuspended in cell culture medium supplemented with 10\% FBS, hydrocortisone, VEGF, EGF, endothelial cell growth factor (ECGS), heparin, L-glutamine, and antibioticantimycotic solution. Cells were seeded into a gelatin-coated flask and incubated in a $5 \% \mathrm{CO}_{2}$ incubator at $37^{\circ} \mathrm{C}$. Culture medium was changed 3 days after the culture start time and then every 2 days (Kalka et al., 2000). To identify the characteristics of BMEPCs, the cells were analyzed with a FACS Aria III cell sorter using APC-conjugated CD133, PE-conjugated CD309, FITCconjugated CD34, FITC-conjugated CD 135, and PE-conjugated CD117 antibodies (eBioscience) (Tian et al., 2019).

Flow cytometric analysis results showed that the majority of the adherent cells displayed both the morphological and qualitative properties of EPCs (Supplementary Figure 2). The cell population positive for CD34 was selected. From the singlet cell population, $72.1 \%$ expressed the marker CD34. Among the CD34-positive cells, 71.4\% expressed the marker CD133, $89.9 \%$ expressed the marker CD309, and $93.9 \%$ expressed the marker CD117.

\section{Hematological and Biochemical Assays}

Blood was collected via cardiac puncture on the 5th, 10th, 15th, and 21st dpi (Supplementary Figure 3). The mice were anesthetized with ketamine (100 $\mathrm{mg} / \mathrm{kg}$, Ilium) and xylazine (10 mg/kg, Ilium) using a previously described process. Blood samples of $400 \mu \mathrm{l}$ were sent for each analysis using an automated hematological and biochemical analyzer.

\section{Histopathology}

After the mice were euthanized, the livers were collected and stored in $10 \%$ formalin. The liver tissue was randomly chosen for sections processing. Microscopic slides were obtained after preparation of wax blocks and staining with hematoxylin and eosin (Leica, IL, United States). Tissue sections were observed under a microscope for differential cell counts. The histopathological sections from each mouse were analyzed quantitatively for the different cell populations percentage, including Kupffer cells, apoptotic cells, mononucleated hepatocytes, and binucleated hepatocytes. The sections were observed under a Nikon Eclipse 50i light microscope, and the image was captured with a Nikon DS-FI1 digital camera. Each slide contained three sections obtained at $15-\mu \mathrm{M}$ intervals, and random six different areas were examined with $3 \times 10^{4} \mu \mathrm{m}$ overlaid grid. The cells were scored with NIS elements D image analysis software (Nikon, New York, NY, United States). Each (mouse) slide was examined with six different areas of $18 \times 10^{4} \mu^{2}$ and each group examined for $54 \times 10^{4}{\mu \mathrm{m}^{2}}^{2}$. The hemorrhagic areas were observed in 20 magnifications (to include a wider area of $216 \times 10^{4} \mu \mathrm{m}^{2}$ per group). All the mean values were analyzed using one-way ANOVA, IBM SPSS statistics version 23, and represented in bar chart.

\section{Immunohistochemistry for DENV 2 Detection}

Paraffin-embedded tissues were sectioned at a thickness of $5 \mu \mathrm{M}$ and prepared on a slide. The mounted sections were deparaffinized at $60^{\circ} \mathrm{C}$ for $2 \mathrm{~h}$ and then immersed into xylene
(HmbG Bendosen, Kuala Lumpur, Malaysia), with two changes at $5 \mathrm{~min}$ each. The slide was then transferred into $100 \%$ ethanol (Fisher Scientific, MA, United States) and incubated for $5 \mathrm{~min}$, followed by $70 \%$ ethanol for $5 \mathrm{~min}$. Next, the slides were immersed into Tris-EDTA buffer $\left(\mathrm{pH} 9.0\right.$ ) for $45 \mathrm{~min}$ at $95^{\circ} \mathrm{C}$ for antigen retrieval. After that, the slides were brought to room temperature, allowed to cool for $20 \mathrm{~min}$, washed with PBS twice for $2 \mathrm{~min}$, and permeabilized with $0.25 \%$ Triton X-100 (Thermo Scientific) in PBS. The slides were then rinsed with PBS, and excess water was blotted from the slide holder for $20 \mathrm{~min}$ at $27^{\circ} \mathrm{C}$. Mouse anti-DENV primary antibodies (ab480914, Acris $\mathrm{GmbH}$, Luzern, Switzerland) were added to the slides at 1:200 dilution. Slides were incubated overnight at $4^{\circ} \mathrm{C}$. The next day, the slides were washed with $\mathrm{PBS}$ at room temperature and further incubated for $20 \mathrm{~min}$ at $37^{\circ} \mathrm{C}$. After that, diluted goat antimouse Alexa Flour 488-conjugated secondary antibody (1:500, BioLegend, San Diego, CA, United States) was added and incubated with each section for $1 \mathrm{~h}$ in a $37^{\circ} \mathrm{C}$ incubator. The slide was further washed with PBS and dried for $15 \mathrm{~min}$ at $37^{\circ} \mathrm{C}$. The slides were then counterstained with $4^{\prime}, 6$-diamidino2-phenylindole (DAPI) at a 1:1,000 dilution for $5 \mathrm{~min}$, rinsed and covered with mounting fluid. The slides were observed under a fluorescence light microscope at $\times 200$ magnification for any positive expression for DENV.

\section{Transmission Electron Microscopy}

The desired area of liver tissues was selected and cut into $1 \mathrm{~mm} \times 1 \mathrm{~mm}$ slices. The tissue was immediately fixed in $3 \%$ glutaraldehyde in $0.1 \%$ phosphate buffer for $4 \mathrm{~h}$, rinsed with phosphate buffer three times for $10 \mathrm{~min}$ each time, and post-fixed with $0.1 \%$ osmium tetroxide in $0.1 \%$ phosphate buffer for $4 \mathrm{~h}$. The tissue was further processed for en bloc staining with $3 \%$ aqueous uranyl acetate in the dark for $1 \mathrm{~h}$ at room temperature. After staining, the specimen was washed, dehydrated, and embedded in resin in a beam capsule. The tissue block was polymerized for $78 \mathrm{~h}$, sectioned into $0.5-1-\mu \mathrm{m}$ sections and dried. These semithin sections were stained with toluidine blue for 2-5 min to select the area of interest. The selected section was trimmed, and ultrathin $70 \mathrm{~nm}$ thick sections were cut with special diamond knives and individually transferred onto a copper grid. The sections were stained with uranyl acetate and lead citrate before viewing under a transmission electron microscope.

\section{RNA Preparation and Sequencing for Transcriptomic Analysis}

RNA sequencing of de novo infected livers was performed with an MiSeq next-generation sequencer (Illumina, Inc., San Diego, CA, United States). Total RNA was extracted from liver tissues using a Qiagen RNeasy Mini kit with in-column DNase treatment as per the manufacturer's instructions (Sessions et al., 2013). RNA concentration and purity were assessed using a NanoDrop 2000c spectrophotometer (NanoDrop Technologies, Wilmington, DE, United States), and RNA integrity was analyzed on a Bioanalyzer 2100 (Agilent Technologies, Santa Clara, CA, United States). A NEBNext Ultra RNA library prep kit for Illumina (New England Biolabs, Ipswich, MA, United States) was used to prepare complementary DNA (cDNA) libraries for RNA sequencing as 
per the manufacturer's instructions. The fragment size, purity, and quantities of the prepared libraries were determined using the Bioanalyzer 2100 (Agilent Technologies). The libraries were then sequenced using a MiSeq reagent kit V3 (Illumina).

\section{Gene Expression (Transcriptomic Analysis)}

Transcriptome assembly and analysis were completed using Tuxedo software. The libraries sequenced were mapped to the updated genomic annotation file of the mouse genome (Mus musculus, NCBI ref. database: GCF_000001635.26_GRCm38.p6_genomic.gff) using Tophat v2.0.9 (Trapnell et al., 2009; Kim et al., 2013). Transcripts were assembled following conformation of reads quality control via the FASTQ format and normalized and quantified by Cufflinks 2.0.2 analysis (Trapnell et al., 2010). Isoforms assembled by Cufflinks from all the sample groups were further merged to create a single transcriptome annotation file in gtf using Cuffmerge. The results were analyzed using Cuffdiff to identify differentially expressed genes and transcripts. The relative expression of genes was determined based on FPKM (total fragments per kilobase of exon per million mapped reads) values. The results obtained were used to generate density and scatter volcano plots using CummeRbund software. Heat maps showing the relative expression of the differentially expressed genes following treatment with stem cells were also generated utilizing the Morpheus heat map generator ${ }^{1}$.

\section{Data Analysis}

The mean values and significant differences were calculated via one-way ANOVA using version 23 IBM SPSS statistics software.

\section{DATA AVAILABILITY STATEMENT}

The data presented in the study are deposited in the (GEO) repository, accession number (GSE175707).

\footnotetext{
${ }^{1}$ https://software.broadinstitute.org/morpheus/
}

\section{REFERENCES}

Akashi, K., Traver, D., Miyamoto, T., and Weissman, I. L. (2000). A clonogenic common myeloid progenitor that gives rise to all myeloid lineages. Nature 404, 193-197. doi: 10.1038/35004599

Baldridge, M. T., King, K. Y., and Goodell, M. A. (2011). Inflammatory signals regulate hematopoietic stem cells. Trends Immunol. 32, 57-65. doi: 10.1016/j. it.2010.12.003

Baldridge, M. T., King, K. Y., Boles, N. C., Weksberg, D. C., and Goodell, M. A. (2010). Quiescent haematopoietic stem cells are activated by IFN-gamma in response to chronic infection. Nature 465, 793-797. doi: $10.1038 /$ nature09135

Barth, O. M., Barreto, D. F., Paes, M. V., Takiya, C. M., Pinhão, A. T., and Schatzmayr, H. G. (2006). Morphological studies in a model for dengue-2 virus infection in mice. Mem. Inst. Oswaldo Cruz 101, 905-915. doi: 10.1590/s007402762006000800014

Basilio-de-Oliveira, C. A., Aguiar, G. R., Baldanza, M. S., Barth, O. M., Eyer-Silva, W. A., and Paes, M. V. (2005). Pathologic study of a fatal case of dengue3 virus infection in Rio de Janeiro, Brazil. Braz. J. Infect. Dis. 9, 341-347. doi: 10.1590/s1413-86702005000400012

\section{ETHICS STATEMENT}

The animal study was reviewed and approved by the Ethical Committee of the IACUC, Universiti Putra Malaysia (ref. no. UPM/IACUC/AUP-R017/2015).

\section{AUTHOR CONTRIBUTIONS}

SK designed the research study, supervised and validated the experiments, edited and finalized the manuscript. SS experimented and contributed in the preparation of stem cells, preparation of DENV2, animal studies, hematological and biochemical assays, histopathology, immunohistochemistry for DENV2 detection, transmission electron microscopy, RNA preparation, data collection and analysis, and manuscript writeup. SP contributed in transmission electron microscopy, hematological and biochemical assays. RM and FB contributed in transcriptomic analysis. PM supervised the preparation of stem cells and edited the manuscript. ST and ZS analyzed data, drafted the manuscript, and prepared the figures. $\mathrm{H}$-YC helped in the propagation of DENV2. RH, GH, BA, CX, NJ, JT, XW, MM, AS, and $\mathrm{AH}$ reviewed the manuscript. All authors contributed to the article and approved the submitted version.

\section{FUNDING}

This study was funded by Deanship of Scientific Research at Jouf University (Research Grant number 40/G/03).

\section{SUPPLEMENTARY MATERIAL}

The Supplementary Material for this article can be found online at: https://www.frontiersin.org/articles/10.3389/fcell.2021. 637270/full\#supplementary-material

Bhatt, S., Gething, P., Brady, O., Messina, J., Farlow, A. W., Moyes, C. L., et al. (2013). The global distribution and burden of dengue. Nature 496:504.

Boelig, M. M., Kim, A. G., Stratigis, J. D., McClain, L. E., Li, H., Flake, A. W., et al. (2016). The Intravenous Route of Injection Optimizes Engraftment and Survival in the Murine Model of In Utero Hematopoietic Cell Transplantation. Biol. Blood Marrow Transplant. 22:991e999.

Brudecki, L., Ferguson, D. A., Yin, D., Lesage, G. D., McCall, C. E., and El Gazzar, M. (2012). Hematopoietic stem-progenitor cells restore immunoreactivity and improve survival in late sepsis. Infect. Immun. 80, 602-611. doi: 10.1128/iai. 05480-11

Dudeck, A., Suender, C. A., Kostka, S. L., von Stebut, E., and Maurer, M. (2011). Mast cells promote Th1 and Th17 responses by modulating dendritic cell maturation and function. Eur. J. Immunol. 41, 1883-1893. doi: 10.1002/eji. 201040994

Franca, R. F., Zucoloto, S., and da Fonseca, B. A. (2010). A BALB/c mouse model shows that liver involvement in dengue disease is immune-mediated. Exp. Mol. Pathol. 89, 321-326. doi: 10.1016/j.yexmp.2010.07.007

Franke, T. (2008). PI3K/Akt: getting it right matters. Oncogene 50:6473. doi: 10.1038/onc.2008.313 
Frascoli, M., and Proietti, M. (2012). F Grassi F. Phenotypic analysis and isolation of murine hematopoietic stem cells and lineage-committed progenitors. J. Vis. Exp. 65:e3736.

Frias-Staheli, N., Dorner, M., Marukian, S., Billerbeck, E., Labitt, R. N., Rice, C. M., et al. (2014). Utility of Humanized BLT Mice for Analysis of Dengue Virus Infection and Antiviral Drug Testing. J. Virol. 88, 2205-2218. doi: 10.1128/jvi. 03085-13

Fujii, H., Hirose, T., Oe, S., Yasuchika, K., Azuma, H., Fujikawa, T., et al. (2002). Contribution of bone marrow cells to liver regeneration after partial hepatectomy in mice. J. Hepatol. 36, 653-659. doi: 10.1016/s0168-8278(02) 00043-0

Geffner, L. F., Santacruz, P., Izurieta, M., Flor, L., Maldonado, B., Auad, A. H., et al. (2008). Administration of autologous bone marrow stem cells into spinal cord injury patients via multiple routes is safe and improves their quality of life: comprehensive case study. Cell Transplant. 17, 1277-1293. doi: 10.3727/ 096368908787648074

Ip, P.-P., and Liao, F. (2010). Resistance to dengue virus infection in mice is potentiated by CXCL10 and is independent of CXCL10-mediated leukocyte recruitment. J. Immunol. 184, 5705-5714. doi: 10.4049/jimmunol.09 03484

Jiang, X., Chen, Y., Wei, H., Sun, R., and Tian, Z. (2013). Characterizing the lymphopoietic kinetics and features of hematopoietic progenitors contained in the adult murine liver in vivo. PLoS One 8:e76762. doi: 10.1371/journal.pone. 0076762

Jope, R. S., Yuskaitis, C. J., and Beurel, E. (2007). Glycogen Synthase Kinase3 (GSK3): inflammation, Diseases, and Therapeutics. Neurochem. Res. 32, 577-595. doi: 10.1007/s11064-006-9128-5

Kalka, C., Masuda, H., and Takahashi, T. (2000). Transplantation of ex vivo expanded endothelial progenitor cells for therapeutic neovascularization. Proc. Natl. Acad. Sci. U. S. A. 97, 3422-3427. doi: 10.1073/pnas.97.7.3422

Kallis, Y. N., Alison, M. R., and Forbes, S. J. (2007). Bone marrow stem cells and liver disease. Gut 56, 716-724. doi: 10.1136/gut.2006.098442

Kim, S., Park, J., Kim, J., Lee, J., and Bang, B. (2013). RNAseq-based transcriptome analysis of Burkholderia glumae quorum sensing. Plant Pathol. J. 29, 249-259. doi: 10.5423/ppj.oa.04.2013.0044

Kroeger, K., Collins, M., and Ugozzoli, L. (2009). The preparation of primary hematopoietic cell cultures from murine bone marrow for electroporation. J. Vis. Exp. 23:1026.

Kuo, C. H., Tai, D. I., Chang-Chien, C. S., Lan, C. K., Chiou, S. S., and Liaw, Y. F. (1992). ). Liver biochemical tests and dengue fever. Am. J. Trop. Med. Hyg. 47, 265-270.

Kuruvilla, J. G., Troyer, R. M., Devi, S., and Akkina, R. (2007). Dengue virus infection and immune response in humanized RAG2 (-/-) gamma(c)(/-) (RAG-hu) mice. Virology 369, 143-152. doi: 10.1016/j.virol.2007. 06.005

Lagasse, E., Connors, H., Al-Dhalimy, H., Reitsma, M., Dohse, M., Osborne, L., et al. (2000). Purified hematopoietic stem cells can differentiate into hepatocytes in vivo. Nat. Med. 6, 1229-1234. doi: 10.1038/81326

Lawn, S. D., Tilley, R., Lloyd, G., Finlayson, C., Tolley, H., Newman, P., et al. (2003). Dengue hemorrhagic fever with fulminant hepatic failure in an immigrant returning to Bangladesh. Clin. Infect. Dis. 37, e1-e4. doi: 10.1086/37 5601

Low, J. S. Y., Wu, K. X., Chen, K. C., Ng, M. M.-L., and Chu, J. J. H. (2011). Narasin, a novel antiviral compound that blocks dengue virus protein expression. Antivir. Ther. 16, 1203-1218. doi: 10.3851/imp1884

Lozito, T., Kolf, C., and Tuan, R. S. (2008). "Microenvironmental regulation of adult mesenchymal stem cells," in Regulatory Networks in Stem Cells, eds V. K. Rajasekhar and M. C. Vemuri (Totowa: Humana Press).

Lozito, T., Kolf, C., and Tuan, R. S. (2009). "Microenvironmental regulation of adult mesenchymal stem cells," in Regulatory Networks in Stem Cells, eds V. K. Rajasekhar and M. C. Vemuri (Totowa: Humana Press), 185-210. doi: 10.1007/ 978-1-60327-227-8_17

Martinez-Gutierrez, M., Correa-Londoño, L. A., Castellanos, J. E., Gallego-Gómez, J. C., and Osorio, J. E. (2014). Lovastatin delays infection and increases survival rates in AG129 mice infected with dengue virus serotype 2. PLoS One 9:e87412. doi: 10.1371/journal.pone.0087412

McAllister, S., Medina, R., O’Neill, C., and Stitt, A. W. (2013). Characterisation and therapeutic potential of endothelial progenitor cells. Lancet 381:S73.
Medina, F., Medina, J. F., Colon, C., Vergne, E., Santiago, G. A., and Munoz-Jordan, J. L. (2012). Dengue virus: isolation, propagation, quantification, and storage. Curr. Protoc. Microbiol. 15:15D.2. doi: 10.1002/9780471729259.mc15d02s27

Mota, J., and Rico-Hesse, R. (2011). Dengue virus tropism in humanized mice recapitulates human dengue fever. PLoS One 6:e20762. doi: 10.1371/journal. pone.0020762

Nagy, R. D., Tsai, B. M., Wang, M., Markel, T. A., Brown, J. W., and Meldrum, D. R. (2005). Stem cell transplantation as a therapeutic approach to organ failure. J. Surg. Res. 129, 152-160. doi: 10.1016/j.jss.2005.04.016

Paes, M. V., Pinhão, A. T., Barreto, D. F., Costa, S. M., Oliveira, M. P., Nogueira, A. C., et al. (2005). Liver injury and viremia in mice infected with dengue-2 virus. Virology 338, 236-246. doi: 10.1016/j.virol.2005.04.042

Paes, M., Lenzi, H., Nogueira, A., Nuovo, G. J., Pinhao, A. T., Mota, E. M., et al. (2009). Hepatic damage associated with dengue-2 virus replication in liver cells of BALB/c mice. Lab. Invest. 89:1140. doi: 10.1038/labinvest.2009.83

Pascutti, M. F., Erkelens, M. N., and Nolte, M. A. (2016). Impact of Viral Infections on Hematopoiesis: from Beneficial to Detrimental Effects on Bone Marrow Output. Front. Immunol. 7:364. doi: 10.3389/fimmu.2016. 00364

Purwati Sumorejo, D. S., Purnama, A Miatmoko, Nasronudin, N., Dinaryanti, A., Karsari, D., et al. (2020). The Potential of Hematopoietic Stem Cells (Hsc) Against Sars-Cov-2 (Covid-19) With Virus Isolates From Indonesia (In Vitro Study). research squares. preprint.

Ruhnke, M., Nussler, A. K., Ungefroren, H., Hengstler, J. G., Kremer, B., and Hoeckh, W. (2005). Human monocyte-derived neohepatocytes: a promising alternative to primary human hepatocytes for autologous cell therapy. Transplantation 79, 1097-1103.

Sakinah, S., Priya, S., Kumari, S., Amira, F., Poorani, K., Alsaeedy, H., et al. (2017). Impact of dengue virus (serotype DENV-2) infection on liver of BALB/c mice: a histopathological analysis. Tissue Cell 49, 86-94. doi: 10.1016/j.tice.2016. 11.005

Salazar Vázquez, B. Y., Cabrales, P., Tsai, A. G., Johnson, P. C., and Intaglietta, M. (2008). Lowering of blood pressure by increasing hematocrit with non nitric oxide scavenging red blood cells. Am. J. Respir. Cell Mol. Biol. 38, 135-142. doi: $10.1165 / \mathrm{rcmb} .2007-00810 \mathrm{C}$

Salter, A. B., Meadows, S. K., Muramoto, G. G., Himburg, H., Doan, P., Daher, P., et al. (2009). Endothelial progenitor cell infusion induces hematopoietic stem cell reconstitution in vivo. Blood 113, 2104-2107. doi: 10.1182/blood-2008-06162941

Samanta, J., and Sharma, V. (2015). Dengue and its effects on liver. World J. Clin. Cases 3, 125-131. doi: 10.12998/wjcc.v3.i2.125

Sekulovic, S., Imren, S., and Humphries, K. (2008). High level in vitro expansion of murine hematopoietic stem cells. Curr. Protoc. Stem Cell Biol. 4:2A.7. doi: 10.1002/9780470151808.sc02a07s4

Seneviratne, S. L., Malavige, G. N., and de Silva, H. J. (2006). Pathogenesis of liver involvement during dengue viral infections. Trans. R. Soc. Trop. Med. Hyg. 100, 608-614. doi: 10.1016/j.trstmh.2005.10.007

Sessions, O. M., Tan, Y., Goh, K. C., Liu, Y., Tan, P., Rozen, S., et al. (2013). Host Cell Transcriptome Profile during Wild-Type and Attenuated Dengue Virus Infection. PLoS Negl. Trop. Dis. 7:e2107. doi: 10.1371/journal.pntd.00 02107

Sreekanth, G. P., Chuncharunee, A., Sirimontaporn, A., Panaampon, J., Noisakran, S., Yenchitsomanus, P.-T., et al. (2016). SB203580 Modulates p38 MAPK Signaling and Dengue VirusInduced Liver Injury by Reducing MAPKAPK2, HSP27, and ATF2 Phosphorylation. PLoS One 11:e0149486. doi: 10.1371/ journal.pone.0149486

Sreekanth, G. P., Chuncharunee, A., Sirimontaporn, A., Panaampon, J., Srisawat, C., Morchang, A., et al. (2014). Role of ERK1/2 signaling in dengue virusinduced liver injury. Vir. Res. 188, 15-26. doi: 10.1016/j.virusres.2014.03. 025

Sreekanth, G. P., Panaampon, J., Suttitheptumrong, A., Chuncharunee, A., Bootkunha, J., Yenchitsomanus, P., et al. (2019). Drug repurposing of N-acetyl cysteine as antiviral against dengue virus infection. Antivir. Res. 166, 42-55. doi: 10.1016/j.antiviral.2019.03.011

Tan, G. K., Ng, J. K., Lim, A. H., Yeo, K. P., Angeli, V., and Alonso, S. (2011). Subcutaneous infection with non-mouse adapted Dengue virus D2Y98P strain induces systemic vascular leakage in AG129 mice. Ann. Acad. Med. Singap. 40, 523-532. 
Taniguchi, E., Kin, M., Torimura, T., Nakamura, T., Kumemura, H., Hanada, S., et al. (2006). Endothelial progenitor cell transplantation improves the survival following liver injury in mice. Gastroenterology 130, 521-531. doi: 10.1053/j. gastro.2005.10.050

Tian, F., Liang, P., and Li, L. (2019). Inhibition of endothelial progenitor cell differentiation by VEGI. Blood 113, 5352-5360. doi: 10.1182/blood-2008-08173773

Trapnell, C., Pachter, L., and Salzberg, S. (2009). TopHat: discovering splice junctions with RNA-Seq. Bioinformatics 25, 1105-1111. doi: 10.1093/ bioinformatics/btp120

Trapnell, C., Williams, B., Pertea, G., and Mortazavi, A. (2010). Transcript assembly and quantification by RNA-Seq reveals unannotated transcripts and isoform switching during cell differentiation. Nat. Biotechnol. 28, 511-515. doi: $10.1038 /$ nbt. 1621

Woolthuis, C. M., and Park, C. Y. (2016). Hematopoietic stem/progenitor cell commitment to the megakaryocyte lineage. Blood 127, 1242-1248. doi: 10.1182/ blood-2015-07-607945

World Health Organization (WHO). (2009). Dengue Guidelines for Diagnosis, Treatment, Prevention and Control: New Edition. Geneva: WHO Press.

Yang, Z., Cai, X., Xu, A., Xu, F., and Liang, Q. (2015). Bone marrow stromal cell transplantation through tail vein injection promotes angiogenesis and vascular endothelial growth factor expression in cerebral infarct area in rats. Cytotherapy 17, 1200-1212. doi: 10.1016/j.jcyt.2015.06.005

Zandi, K., Lani, R., Wong, P., Teoh, B., Sam, S., and Johari, J. (2012). Flavone enhances dengue virus type-2 (NGC strain) infectivity and replication in Vero cells. Molecules 17, 2437-2445. doi: 10.3390/molecules 17032437
Zellweger, R. M., Cano, J., Mangeas, M., Taglioni, F., Mercier, A., Despinoy, M., et al. (2017). Socioeconomic and environmental determinants of dengue transmission in an urban setting: an ecological study in Nouméa, New Caledonia. PLoS Negl. Trop. Dis. 11:e0005471. doi: 10.1371/journal.pntd. 0005471

Zhang, Y., Bai, X.-F., and Huang, C.-X. (2003). Hepatic stem cells: existence and origin. World J. Gastroenterol. 9, 201-204. doi: 10.3748/wjg.v9.i2.201

Zheng, J., Song, C., and Zhang, C. C. (2011). A new chapter: hematopoietic stem cells are direct players in immunity. Cell Biosci. 1:33. doi: 10.1186/2045-3701$1-33$

Conflict of Interest: The authors declare that the research was conducted in the absence of any commercial or financial relationships that could be construed as a potential conflict of interest.

The reviewer RR declared a shared affiliation, with no collaboration, with one of the authors, $\mathrm{AH}$, to the handling editor at the time of the review.

Copyright (c) 2021 Sakinah, Priya, Mok, Munisvaradass, Teh, Sun, Alzahrani, Abu Bakar, Chee, Awang Hamat, He, Xiong, Joseph, Tong, Wu, Maniam, Samrot, Higuchi and Kumar. This is an open-access article distributed under the terms of the Creative Commons Attribution License (CC BY). The use, distribution or reproduction in other forums is permitted, provided the original author(s) and the copyright owner(s) are credited and that the original publication in this journal is cited, in accordance with accepted academic practice. No use, distribution or reproduction is permitted which does not comply with these terms. 